\title{
Quilting Effect and Appearance Change according to Fabric Properties and Surface Reconstruction Method based on 3D Digital Clothing System ${ }^{+}$
}

\author{
Yoon Jihae $\cdot$ Kim Jongjun ${ }^{*}$ \\ Dept. of Clothing and Textiles, Graduate School, Ewha Womans University, Master Course \\ Dept. of Clothing and Textiles, Ewha Womans University, Professor
}

\begin{abstract}
Quilting, a technique to join two or more layers of fabrics, has long been used in the textile and fashion sectors. To evaluate dimensional effect of quilting that changes according to the characteristics of fabrics, 30 scanning method is employed in this study. Goal of this study is to interpret how fabric's composition, stiffness, thickness, and weight affect the appearance when quilted fabrics are used in a garment. Surface reconstruction method based on 3D scanning is used as a research method to evaluate the changing appearance depending on the material properties quantitatively with the quilting method. Besides, exemplary virtual clothing is realized through a virtual quilting method in $3 \mathrm{D}$ digital clothing system based on the properties of fabrics.
\end{abstract}

Key Words : Quilting, Stiffness, 3D Reconstruction, Drape, 3D Scanning Surface Image

\section{Introduction}

Along with the recent development of $3 D$ CAD technologies, the application scope of 3-dimensional CAD is becoming greater in the textile and fashion industries. The fashion material design $C A D$ and $3 D$ digital clothing $C A D$ systems are excellent tools in reducing working hours with easier adjustment and stream-lined producing procedures as well as maximizing efficiency, consequently being able to elevate overall productivity. For these reasons, these systems are getting popular in related companies and industrial sections. ${ }^{1)}$

As researches in textile and fashion industries and technologies of IT field tend to get combined these days, the textile and fashion industries and academic fields are also actively

${ }^{+}$The contents of this paper will be included in the master's thesis to be published.

Corresponding author: Kim Jongjun, Tel.+82-2-3277-3102 Fax.+82-2-2179-8480

E-mail: jjkim@ewha.ac.kr 
Yoon Jihae $\cdot$ Kim Jongjun / Quilting Effect and Appearance Change according to Fabrics Properties and Surface Reconstruction Method based on 3D Digital Clothing System

adopting the $3 D$ digital clothing CAD system.

Interlining, a very important part of a garment, is a fabric inserted into clothing in order to form and maintain silhouette of the outfit. Various materials such as hemp cloth, wool, cotton and felt can be used as interlining. Characterized with thickness, the interlining keeps the outline of garment with its stiffness and elasticity. Since the interlining is a functional material used inside of garment, it is hard to find various colors and patterns in interlining fabrics. Due to the limited colors and patterns, it is generally not desirable to use it for exterior layer of apparel. Notwithstanding such general circumstances, this study aims to increase dimensional and aesthetic effect of interlining fabrics, as exterior material, using the quilting method that adds decorative effect to it. The quilting is the highlight texture of fabric surface with its dimensional effect of stitch lines composed by sewing machine or handmade. For various and decorative effect, the quilting method has been consistently used in the fashion industry. As decorated pattern in material surface with quilting stitches highlights the garment fabric, the quilting method is used as a significant decorative element in fashion industry to maximize splendidness and aesthetic effect of outfit. $^{2)}$ Furthermore, inserting cotton or polyester wadding between the layers of fabrics can bring in additional dimensional and thermal effects. Insulating layer formed by in-between cotton or polyester wadding provides thermal effect.

This study aims to interpret how exterior is affected by fabric's composition, structure, stiffness, thickness, and weight by applying the quilting method to the interlining and comparison materials. The surface reconstruction method with $3 D$ scanning $^{3)}$ is employed as a research method to evaluate the changing exterior depending on the materials quantitatively. Considering the property of the fabric, an apparel is realized using a virtual quilting method in $3 D$ digital clothing system ${ }^{4}$. It aims to provide data about fashion materials so that the textile and fashion industries can develop various high-value products by using the $3 D$ virtual apparel realization method of CAD software system.

\section{Experimental Methods}

\section{Fabric Specimen Selection}

Fabric materials were selected as specimens to apply the quilting method. Among various fabrics, denim and organza with high stiffness and big difference in weight per unit area were chosen to be compared with two different kinds of interlining. In the following specimen data, $1-1$ stands for interlining with small amount of hair, and $1-2$ for interlining with large amount of hair, $D$ for denim, $O$ for organza and $P$ for polyester wadding used in quilting. Basic specifications of specimens such as fiber composition and structure are shown in <Table $1>$

\section{Stiffness Measurement}

Fabric stiffness measuring test was carried on adhering to 'Fabric Flexibility Measuring Method: Flexometer (KS-K0539)'. 3 types of specimens with 4 different fabrics for each specimen were prepared for this test as shown in 〈Fig. 1>. Type $A$ is $2.5 \times 15.0 \mathrm{~cm}$ (warp and weft) size of rectangular shape fabric, Type $B$ is in uniform size of the former with one seam in the center, and Type $C$ is $2.5 \times 20.0 \mathrm{~cm}$ and pad-inserted and stitched. These specimens were laid on a flat 
$<$ Table 1> Specification of Specimens.

\begin{tabular}{|c|c|c|c|c|c|c|c|}
\hline \multirow{2}{*}{ Fabric } & \multirow{2}{*}{$\begin{array}{l}\text { Weight } \\
\left(\mathrm{g} / \mathrm{m}^{2}\right)\end{array}$} & \multicolumn{2}{|c|}{ Fiber composition } & \multicolumn{2}{|c|}{ Yarn Count } & \multicolumn{2}{|c|}{ Fabric Count } \\
\hline & & Warp(\%) & Filling(\%) & Warp & Filling & Warp & Filling \\
\hline $\mid-1$ & 186.0 & Cotton 100 & $\begin{array}{r}\text { Polyester } 25.6 \text {, Rayon } \\
39.0 \text {, Hair } 35.4 \\
\end{array}$ & $19.8 \mathrm{Ne}$ & $1 / 10.4 \mathrm{Nm}$ & 40 & 40 \\
\hline $1-2$ & 215.0 & Cotton 100 & Rayon 29.8, Hair 70.2 & $20.2 \mathrm{Ne}$ & $1 / 11.0 \mathrm{Nm}$ & 46 & 46 \\
\hline D & 464.0 & Cotton 100 & Cotton 100 & 7.1 $\mathrm{Ne}$ & $6.1 \mathrm{Ne}$ & 66 & 46 \\
\hline 0 & 37.0 & $\begin{array}{c}\text { Polyester } \\
100 \\
\end{array}$ & Polyester 100 & $44.2 \mathrm{D}$ & $43.2 \mathrm{D}$ & 94 & 87 \\
\hline$P$ & 92.8 & \multicolumn{2}{|c|}{ Polyester 100} & \multicolumn{2}{|c|}{ - } & \multicolumn{2}{|c|}{ - } \\
\hline
\end{tabular}

platform and pushed toward warp plane slowly and then measured bending length of the specimen when fabric specimen touched the warp plane. In the same way, surface and back side were also measured and recorded. When warp plane and horizontal line of specimen did not go parallel due to the bending, the center of bent part was rearranged to match with the warp plane and measured again. Drape stiffness $(C, \mathrm{~cm})$ was $L / 2$ and Flex stiffness(G, $\mathrm{cm} \cdot \mathrm{gf})$ was $\mathrm{W} \times \mathrm{C}^{3} . \mathrm{W}$ indicates weight of specimen per unit area $\left(\mathrm{gf} / \mathrm{cm}^{2}\right)$. The stiffness measurement value of the warp or weft direction is designated as _ 1 or _ 2 for each specimen. For example, $1-1 \_1$ indicates warp direction of specimen $1-1$.

\section{Weight}

3 pieces of $10 \times 10$ square-shaped specimens were measured and their mean values were used as weight.

\section{Analysis based on KES-FB System ${ }^{5}$}

sln order to examine dynamic properties of each specimen, their tensile, bending, shear, surface, compression and thickness were measured with KES-FB system.

\section{Structured light 3D Scanning}

Each fabric specimen was cut into $2 \times 15 \mathrm{~cm}$ size, then the fabric specimens and stitched specimens were scanned with a 3D scanner ${ }^{6)}$ using stiffness measuring equipments. The data were arranged in a screen with Meshlab ${ }^{7)}$. The obtained coordinates were moved parallel in Rhino3D ${ }^{8)}$ and new specimen surface coordinates were drawn with 'PolylinePoints' function. These data were reconstructed into dimensional coordinates in Excel program. Sleeve specimen of intended outfit was produced and quilt specimen was charted in the same way.

\section{3D Scanning by Reconstruction Program and RGB-D Camera}

30 scanning was conducted to measure draping exterior of sleeve specimen when hung on human body. It was recorded with an RGB-D 
Yoon Jihae $\cdot$ Kim Jongjun / Quilting Effect and Appearance Change according to Fabrics Properties and Surface Reconstruction Method based on 3D Digital Clothing System

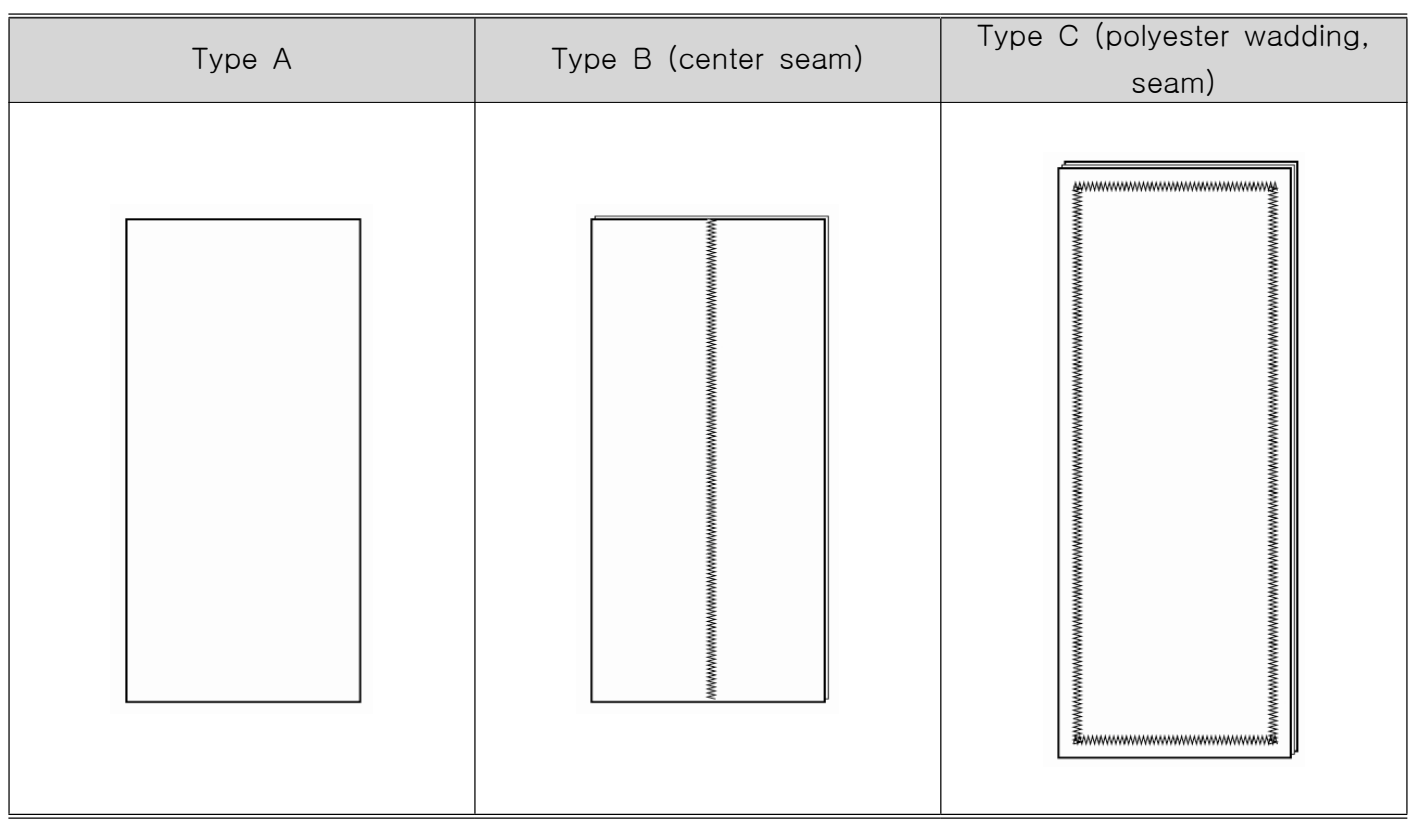

<Fig. 1> Types of Fabric Specimen Preparation.

(Microsoft Kinect ${ }^{9)}$ ) camera using a reconstruction program (RecontructMe ${ }^{10)}$ ), and arranged with MeshLab. These were saved in a STL file format and then the coordinates were moved parallel in Rhino3D. The dimensional coordinates in sleeve specimen were drawn with 'PolylinePoints' function and reconstructed to a chart.

\section{3D Digital Clothing CAD Program}

CLO $3 D$ is a $3 D$ digital clothing CAD system that produces intended image by combining $3 D$ virtual technologies into fashion industry and digitalizing sample producing procedure. Scanned 4 kinds of different fabrics were applied and intended patterns were designed with the quilting method in the program. Then, realized drapes of the materials and profiles with the simulated results were observed and compared.

\section{Results and Discussion}

\section{Stiffness analysis}

According to the stiffness test conducted by Flexometer to measure bending length of specimen contacting the warp plane, 1-2_2 interlining weft) among the specimens(Type A) shows the highest value, which means the highest stiffness as shown in <Fig. 2>. This is assumed due to the highest percentage of hair fiber. 1-2_1 (interlining warp) is the most flexible. In the stitched specimens(Type B), D-s(Denim) is the stiffest and I-2s_1(interlining warp) is the most flexible. Generally, stitched specimens are stiffer than not-stitched fabric specimens. This is due to the fact that changes of two-layered fabric specimens around the stitched spot cause binding effect. In the pad-inserted and stitched specimens(Type C), I-2sp_2(interlining weft) is the most stiff and $1-2 \mathrm{sp}_{-} 1$ (interlining warp) is 
the most flexible. This Type $C$ specimens have extraordinarily high stiffness compared to other specimens(Types A and B). It is assessed due to the effect of stitching and polyester wadding.

\section{Weight}

Pad is inserted into $10 \times 10$ size of square-shaped specimens and stitched. These specimens were measured 3 times and the average values were recorded as shown in <Fig. 3>. Also, sleeve samples of intended outfit was

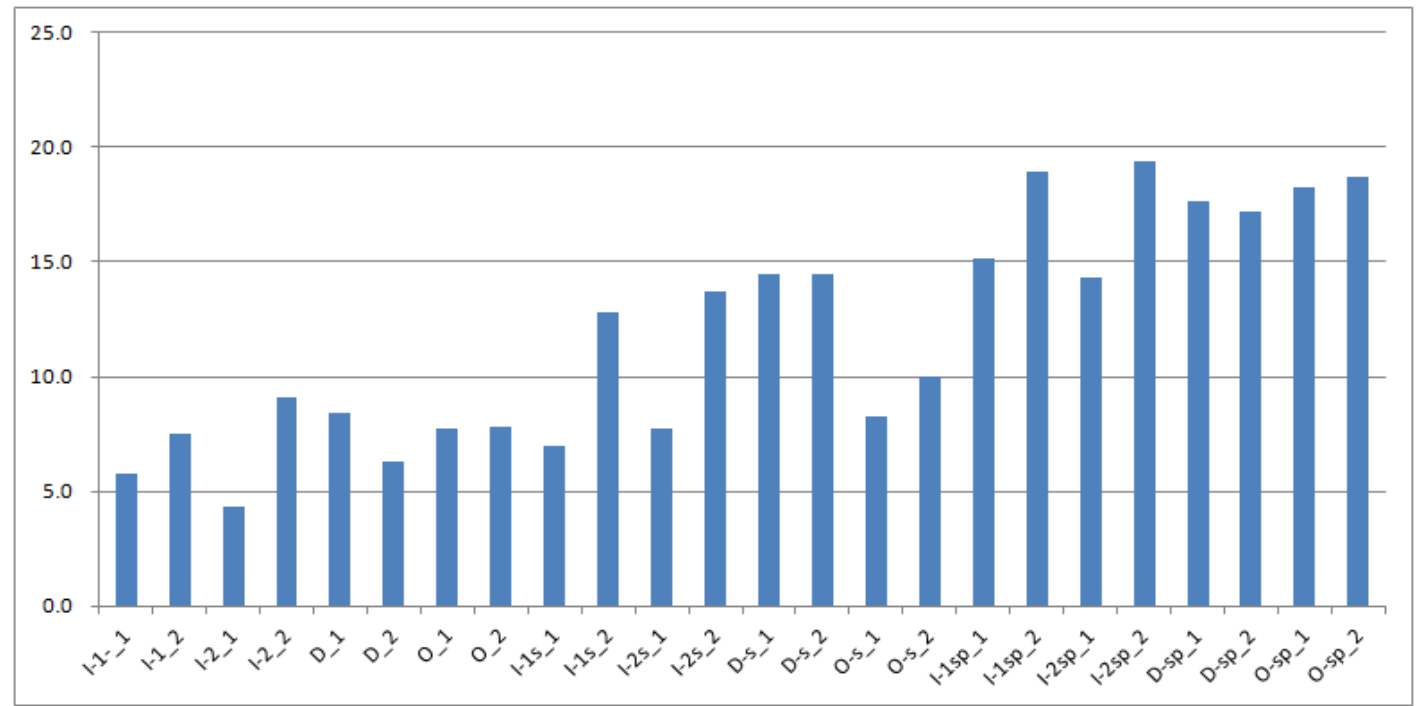

<Fig. 2> Bending length(L, cm).

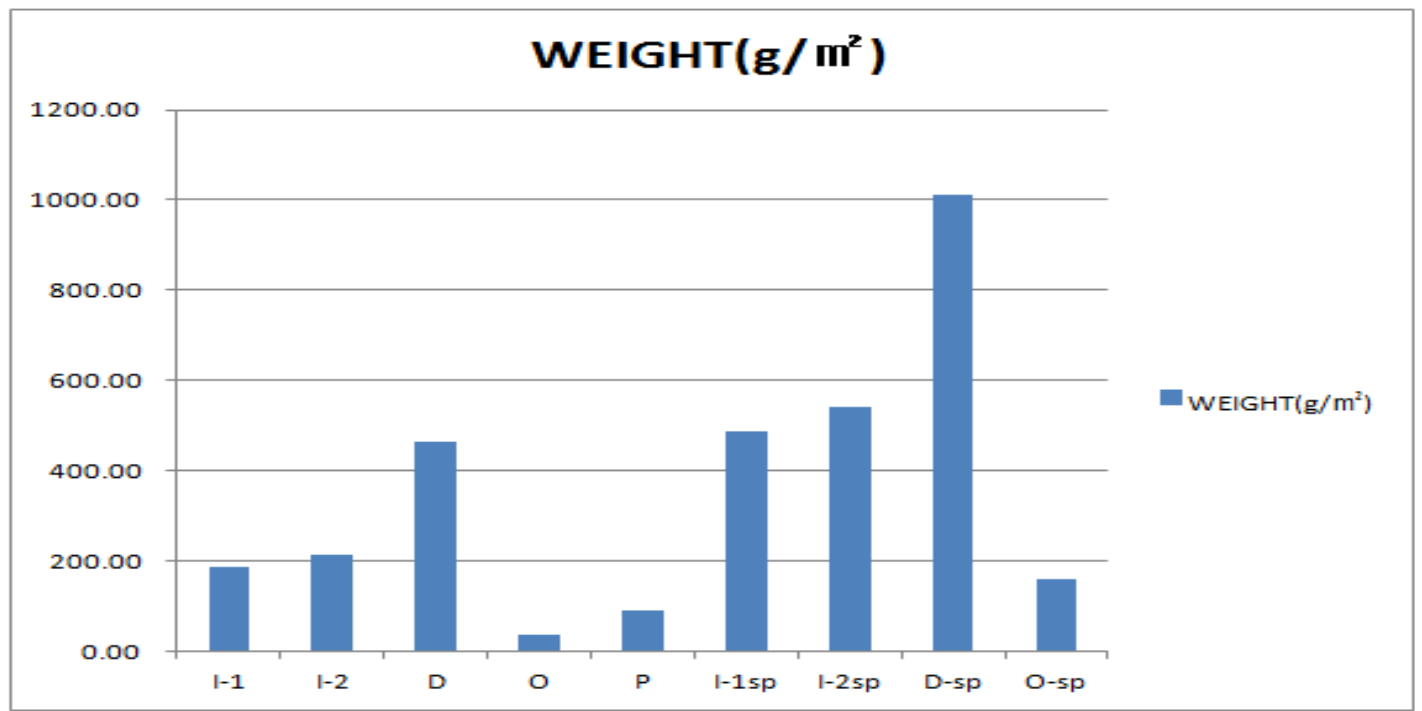

<Fig. 3> Weight of fabric specimens(Type $\mathrm{A}$ and $\mathrm{C}$ ). 
Yoon Jihae $\cdot$ Kim Jongjun / Quilting Effect and Appearance Change according to Fabrics Properties and Surface Reconstruction Method based on 3D Digital Clothing System

$<$ Table 2> Weight of Sleeve Sample.

\begin{tabular}{c|c}
\hline \hline Sleeve & Weight $(\mathrm{g})$ \\
\hline I-1-Sv & 49.02 \\
\hline I-2-Sv & 52.12 \\
\hline D-Sv & 81.93 \\
\hline O-Sv & 24.70 \\
\hline \hline
\end{tabular}

<Table 3> Physical Mechanical Property Measurement with KES-FB.

\begin{tabular}{|c|c|c|c|c|c|c|}
\hline \multicolumn{2}{|c|}{ ITEM } & $1-1$ & $1-2$ & $D$ & 0 & UNIT \\
\hline \multirow{8}{*}{ TENSILE } & EM1 & 5.93 & 13.32 & 8.10 & 2.59 & \multirow{4}{*}{$\%$} \\
\hline & EM2 & 2.64 & 1.68 & 5.51 & 1.88 & \\
\hline & LT1 & 0.766 & 0.715 & 0.886 & 0.880 & \\
\hline & LT2 & 0.765 & 0.738 & 0.918 & 0.926 & \\
\hline & WT1 & 11.35 & 23.80 & 17.95 & 5.70 & \multirow{2}{*}{ None } \\
\hline & WT2 & 5.05 & 3.10 & 12.65 & 4.35 & \\
\hline & RT1 & 53.74 & 45.80 & 44.85 & 71.05 & af. $\mathrm{cm} / \mathrm{cm}^{2}$ \\
\hline & RT2 & 54.46 & 54.84 & 41.90 & 72.41 & 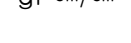 \\
\hline \multirow{4}{*}{ BENDING } & $\mathrm{B} 1$ & 0.230 & 0.099 & 0.798 & 0.159 & \multirow{2}{*}{$\mathrm{gf} \cdot \mathrm{cm}^{2} / \mathrm{cm}$} \\
\hline & B2 & 0.983 & 1.069 & 0.572 & 0.147 & \\
\hline & $2 \mathrm{HB} 1$ & 0.0983 & 0.0782 & 0.7332 & 0.0229 & af. $\mathrm{cm} / \mathrm{cm}$ \\
\hline & $2 \mathrm{HB} 2$ & 0.3249 & 0.3995 & 0.6418 & 0.0272 & g) . Cin/ Cin \\
\hline \multirow{6}{*}{ SHEARING } & G1 & 1.03 & 1.14 & 5.97 & 0.34 & \multirow{6}{*}{$\mathrm{gf} \cdot \mathrm{cm} \cdot \mathrm{deg}$} \\
\hline & G2 & 1.04 & 1.24 & 5.43 & 0.24 & \\
\hline & $2 \mathrm{HG} 1$ & 1.13 & 1.78 & 13.10 & 0.13 & \\
\hline & $2 \mathrm{HG} 2$ & 1.00 & 2.03 & 12.8 & 0.08 & \\
\hline & 2HG51 & 3.53 & 4.45 & 17.38 & 0.63 & \\
\hline & 2HG52 & 3.48 & 4.88 & 15.83 & 0.48 & \\
\hline \multirow{6}{*}{ SURFACE } & MIU1 & 0.192 & 0.223 & 0.333 & 0.123 & \multirow{4}{*}{ None } \\
\hline & MIU2 & 0.18 & 0.228 & 0.349 & 0.156 & \\
\hline & MMD1 & 0.0560 & 0.0507 & 0.0193 & 0.0316 & \\
\hline & MMD2 & 0.05 & 0.0438 & 0.0332 & 0.0458 & \\
\hline & SMD1 & 20.00 & 20.00 & 5.07 & 7.34 & \multirow{2}{*}{$\mu \mathrm{m}$} \\
\hline & SMD2 & 16.47 & 15.51 & 8.41 & 8.07 & \\
\hline \multirow{3}{*}{ COMPRESSION } & LC & 0.348 & 0.340 & 0.380 & 0.737 & None \\
\hline & WC & 0.134 & 0.108 & 0.264 & 0.035 & $\mathrm{gf} \cdot \mathrm{cm} / \mathrm{cm}^{2}$ \\
\hline & $\mathrm{RC}$ & 55.22 & 48.15 & 43.56 & 65.71 & $\%$ \\
\hline THICKNESS & $\mathrm{T}$ & 0.657 & 0.637 & 1.277 & 0.156 & $\mathrm{~mm}$ \\
\hline WEIGHT & W & 18.355 & 20.670 & 44.490 & 3.625 & $\mathrm{mg} / \mathrm{cm}^{2}$ \\
\hline
\end{tabular}


produced and measured their weight as shown in <Table 2>. According to the measuring of fabric specimens and pad-inserted specimens weights, they turn out to be in the order of $D$ $>\mid-2>1-1>0$ and sleeve sample weights are in the order of $\mathrm{D}-\mathrm{Sv}>1-2-\mathrm{Sv}>1-1-\mathrm{Sv}>$ O-Sv.

\section{Specimens' Physical Mechanical Property Measurement with KES-FB}

In order to find dynamic properties of each specimen, their tensile, bending, shear, surface, compression, thickness and weight were measured with KES-FB system. The test measured $1-1, \mid-2, D, O$ of 4 specimens and the results are shown in <Table 3$\rangle$.

In tensile property, EM1(warp direction) is in order of $|-2>D>|-1>0$ and EM2 (weft direction) is $D>|-1>0>|-2$. High extension ratio of specimen when adding maximum load means easy extension. In bending property, $B 1$ is in the order of $\mathrm{D}>|-1>0>|-2$ and $\mathrm{B} 2$ is $|-2>|-1>D>0$. Hair fiber has markedly high rigidity compared to other general textiles. Hair fiber and ordinary textiles are mixed often so as to maximize stiffness of the interlining. Looking at the weft composition of specimens as described in $\langle$ Table 1>, I-2 contains $70 \%$ of hair fiber and $1-1$ does $35 \%$. Filling fabric count of $1-2$ is higher than that of $1-1$. Therefore $1-2$ shows higher bending rigidity in weft. In shear rigidity, G1 is in the order of $D>|-2>|-1>0$. As $D$ (Denim) has a high shear rigidity, its adaptability to the curvy line of human body is assessed to be relatively low. Thickness $(\mathrm{mm})$ is in order of $D>|-1>|-2>0$. Mean values of KES-measured warp and weft directions are shown in $\langle$ Table 4$\rangle$.

$<$ Table $4>$ Mean values of KES measurement

\begin{tabular}{|c|c|c|c|c|c|c|}
\hline \multicolumn{2}{|c|}{ ITEM } & $1-1$ & $1-2$ & $D$ & 0 & UNIT \\
\hline \multirow{4}{*}{ TENSILE } & EM & 4.28 & 7.50 & 6.81 & 2.23 & \multirow{2}{*}{$\%$} \\
\hline & LT & 0.765 & 0.726 & 0.902 & 0.903 & \\
\hline & WT & 8.20 & 13.45 & 15.30 & 5.02 & None \\
\hline & $\mathrm{RT}$ & 54.10 & 50.32 & 43.38 & 71.73 & $\mathrm{~g} \cdot \mathrm{cm} / \mathrm{cm}^{2}$ \\
\hline \multirow{2}{*}{ BENDING } & $B$ & 0.607 & 0.584 & 0.685 & 0.153 & $\mathrm{gf} \cdot \mathrm{cm}^{2} / \mathrm{cm}$ \\
\hline & $2 \mathrm{HB}$ & 0.2116 & 0.2388 & 0.6875 & 0.0250 & $\mathrm{gf} \cdot \mathrm{cm} / \mathrm{cm}$ \\
\hline \multirow{3}{*}{ SHEARING } & $G$ & 1.03 & 1.19 & 5.70 & 0.29 & $\mathrm{~g} \cdot \mathrm{cm} \cdot \mathrm{deg}$ \\
\hline & $2 H G$ & 1.07 & 1.90 & 12.95 & 0.10 & \multirow{2}{*}{$\mathrm{gf} / \mathrm{cm}$} \\
\hline & $2 \mathrm{HG} 5$ & 3.51 & 4.66 & 16.60 & 0.56 & \\
\hline \multirow{3}{*}{ SURFACE } & MIU & 0.188 & 0.226 & 0.341 & 0.140 & \multirow{2}{*}{ None } \\
\hline & MMD & 0.0536 & 0.0473 & 0.0258 & 0.0387 & \\
\hline & SMD & 18.24 & 17.76 & 6.74 & 7.71 & $\mu \mathrm{m}$ \\
\hline \multirow{3}{*}{ COMPRESSION } & LC & 0.348 & 0.340 & 0.380 & 0.737 & None \\
\hline & WC & 0.134 & 0.108 & 0.264 & 0.035 & $\mathrm{gf} \cdot \mathrm{cm} / \mathrm{cm}^{2}$ \\
\hline & $\mathrm{RC}$ & 55.22 & 48.15 & 43.56 & 65.71 & $\%$ \\
\hline THICKNESS & $T$ & 0.657 & 0.637 & 1.277 & 0.156 & $\mathrm{~mm}$ \\
\hline WEIGHT & W & 18.355 & 20.670 & 44.490 & 3.625 & $\mathrm{mg} / \mathrm{cm}^{2}$ \\
\hline
\end{tabular}


Yoon Jihae $\cdot$ Kim Jongjun / Quilting Effect and Appearance Change according to Fabrics Properties and Surface Reconstruction Method based on 3D Digital Clothing System

\section{Stiffness Test of Fabric Specimens and Stitched Specimens}

The draping shape of 4 specimens and stitched specimens were analyzed with stiffness measuring device using Flexmeter and $3 D$ scanner. The results are shown in <Fig. 4 7>. For comparative analysis of the specimens; a method to push certain length of the specimens was employed.

The result of pushing away the specific length
$(6.6 \mathrm{~cm})$ from the Flexometer specimen line is as in the followings.

According to the <Fig. 4> with the results of warp direction of the specimens, their drapes are in the order of $D>0>|-1>|-2$. However, when it comes to weft, the order changes to $|-2>0>|-1>D$ as depicted in <Fig. 5>. It is evaluated that the factors of $B$, the result of KES-FB, and fabric weight influence to the drape of the specimens as described in <Table $4>$.

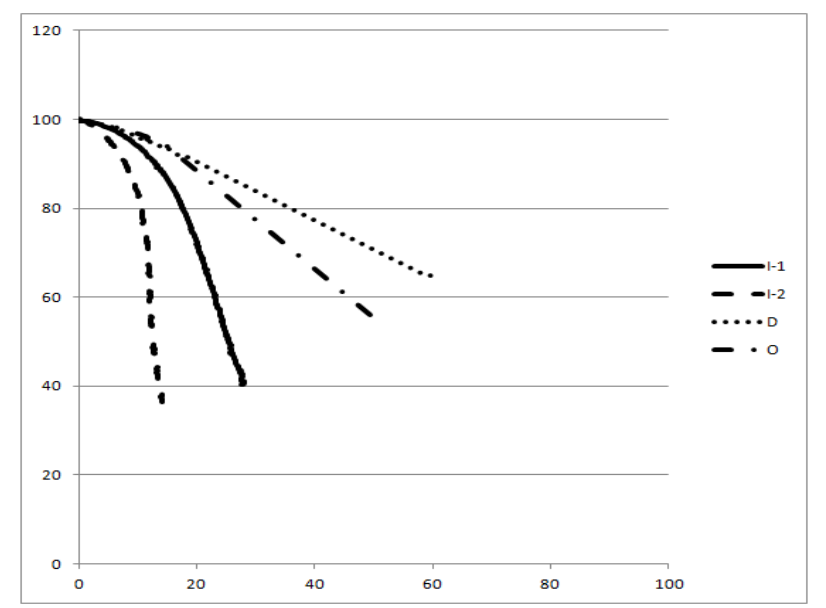

<Fig 4.> Bending profile of $1-1,1-2, D, O$ specimens, warp $6.6 \mathrm{~cm}$.

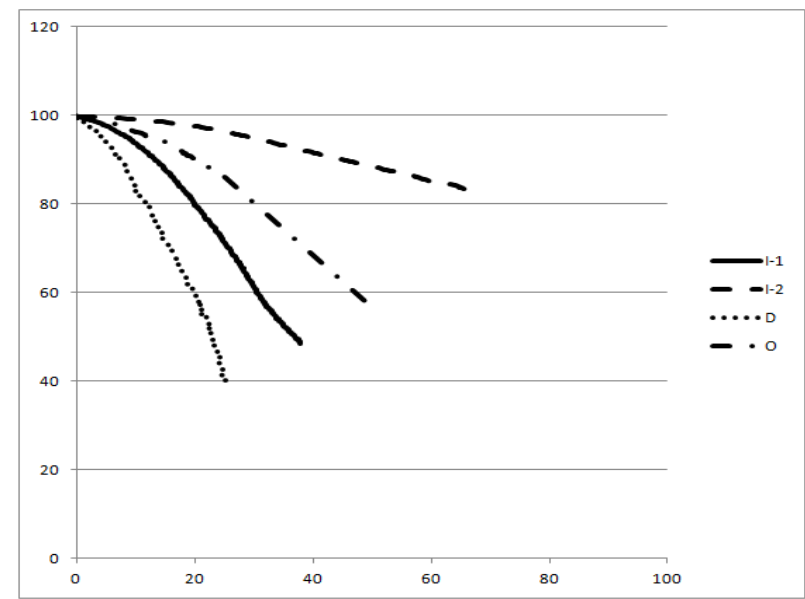

<Fig. 5> Bending profile of $\mid-1,1-2, D, O$ specimens, filling $6.6 \mathrm{~cm}$. 
Results of the stitched specimens (Type B) are as follows. The warp drapes in the order of $D>0>|-2 \simeq|-1<$ Fig. 6> while the weft drapes in the order $\mathrm{D}\rangle|-2\rangle|-1\rangle 0\langle$ Fig. 7$\rangle$ it is concluded that the order of specimens are changing because being composed of 2 different fabrics.

According to the above result, the dimensional coordinates obtained by $3 \mathrm{D}$ scanning can be used to analyze the drapes of fabric specimens. Such precise dimensional analysis suggests that not only flat specimen but also complicatedly- formed or dimensionally processed specimens can be analyzed. Even when it is difficult to measure them due to axis contortion, it is possible to obtain precise information through this method.

\section{Quilting Profile Analysis of quilted Sleeve Specimen Surface}

Actual sleeve specimen for the intended apparel was produced and scanned with $3 \mathrm{D}$

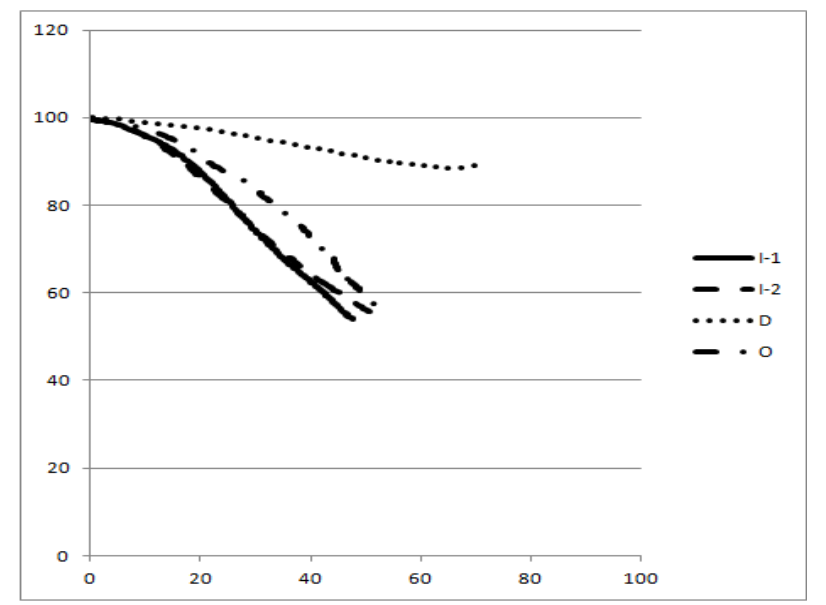

<Fig. 6> Bending profile of $1-1,1-2, \mathrm{D}, \mathrm{O}$ stitched specimens, warp $6.6 \mathrm{~cm}$.

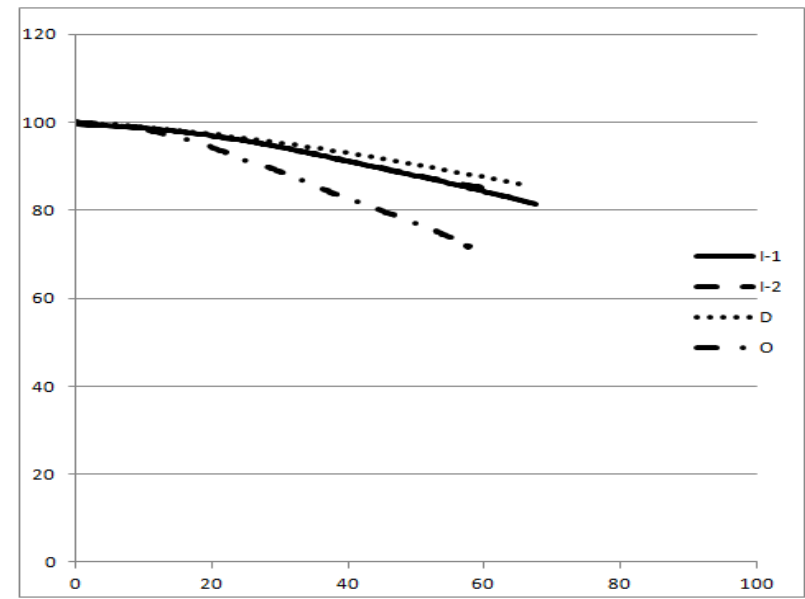

<Fig. 7> Bending profile of $1-1,1-2, D, 0$ stitched specimens, filling $6.6 \mathrm{~cm}$. 
Yoon Jihae $\cdot$ Kim Jongjun / Quilting Effect and Appearance Change according to Fabrics Properties and Surface Reconstruction Method based on 3D Digital Clothing System

scanner(Structured light) to see the exterior of each material changed by quilting. The obtained data was arranged in a screen with MeshLab and saved in a STL file. These were moved parallel and then, coordinates with Rhino3D and dimensional coordinates of sleeve surface were drawn using 'PolylinePoints' function. <Fig. 8> The 3 mountain shapes around peaks are caused by polyester wadding and quilting and the valley shaped are by compressed polyester wadding due to stitching. With this information, the effect of quilting can be dimensionally analyzed. That is to say, height of the peaks can be calculated by drawing vertical line from the highest point to the base line that connects valley to valley.
Mean height of each specimen's quilting is recorded in <Table 5> Specimen $\mathrm{O}(3.46 \mathrm{~mm})$ shows the most distinct dimensional effect of the quilting. It seems because the $O$ specimen has low stiffness and shear rigidity so that the polyester wadding and quilting effect appears dramatically. On the other hand, D specimen $(2.13 \mathrm{~mm})$ is thick $(1.28 \mathrm{~mm})$ with high shear rigidity and stiffness so that quilting does not bring clear dimensional effect.

\section{Photomicrographs}

Specimens were photographed with microscope in order to analyze their status. 10x magnification of eyepiece and $0.7 x, 2.0 x, 3.0 x$ of objective lens are shown in $\langle$ Table 6$\rangle$ As 0 specimen
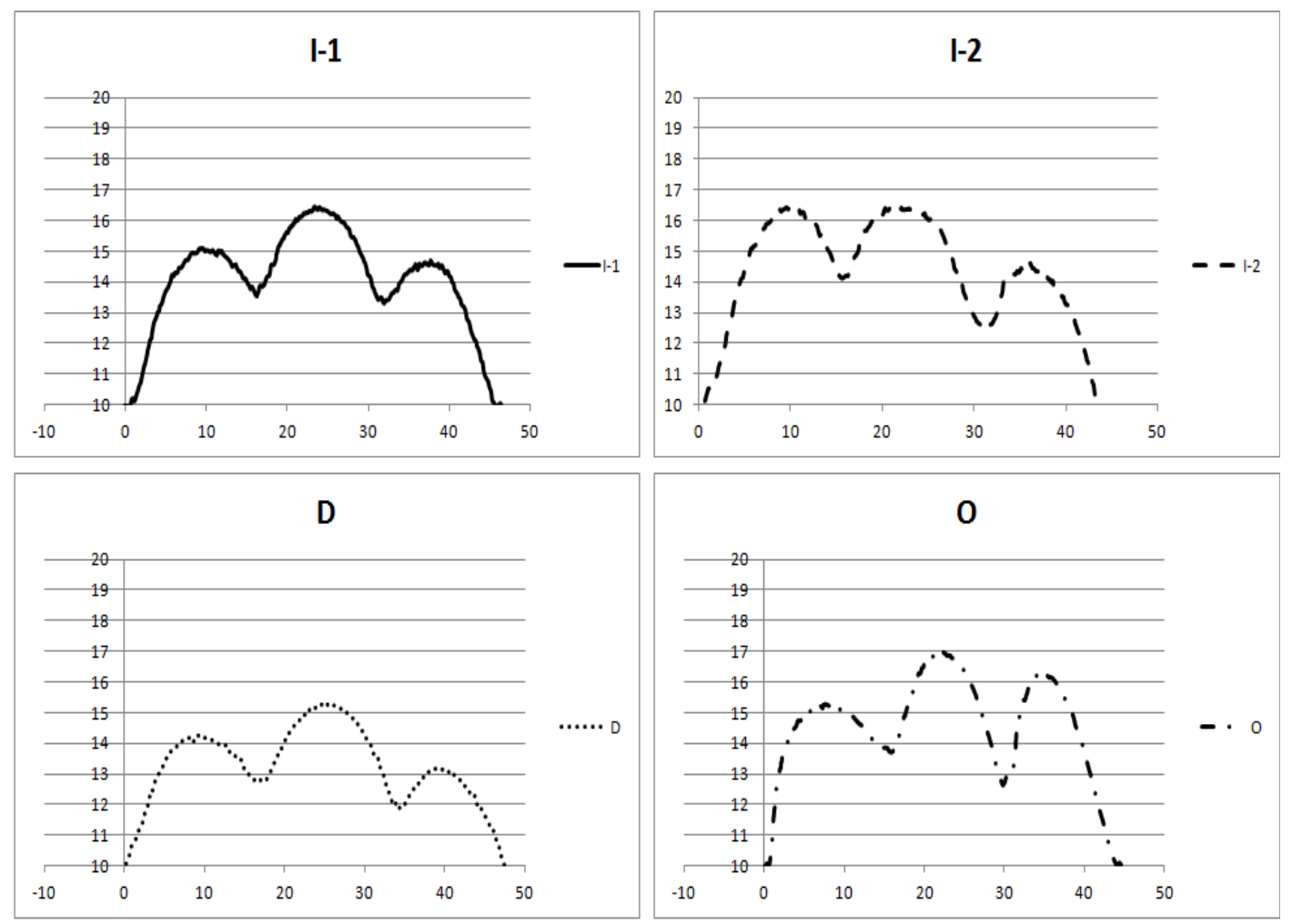

<Fig. 8> Height profile of the surface of $3 D$ scanned objects. 
has low density between warp and weft and is composed of slick filament, shear transformation can be easily occurred.

\section{Analysis on Drape of quilted Sleeve Specimen Mounted on a Body}

In oder to observe drape shape of the quilted sleeve specimen, each specimen was mounted on a same mannequin's shoulder. An RGB-D sensor was used for this test to interpret the general shape including the specimens and the mannequin in 3-dimensional method. Also, the specimens were scanned using the ReconstructMe software, a 30 scanning and reconstruction program, and their coordinate values were saved. Selected two specimens, I-1 and $\mathrm{O}$, were arranged on a screen prepared by MeshLab.<Fig. 9> Each quilted sleeve specimen shows different three-dimensional drape. Complicated

$<$ Table 6> Photomicrographs of fabric specimens (eyepiece mag. 10x)

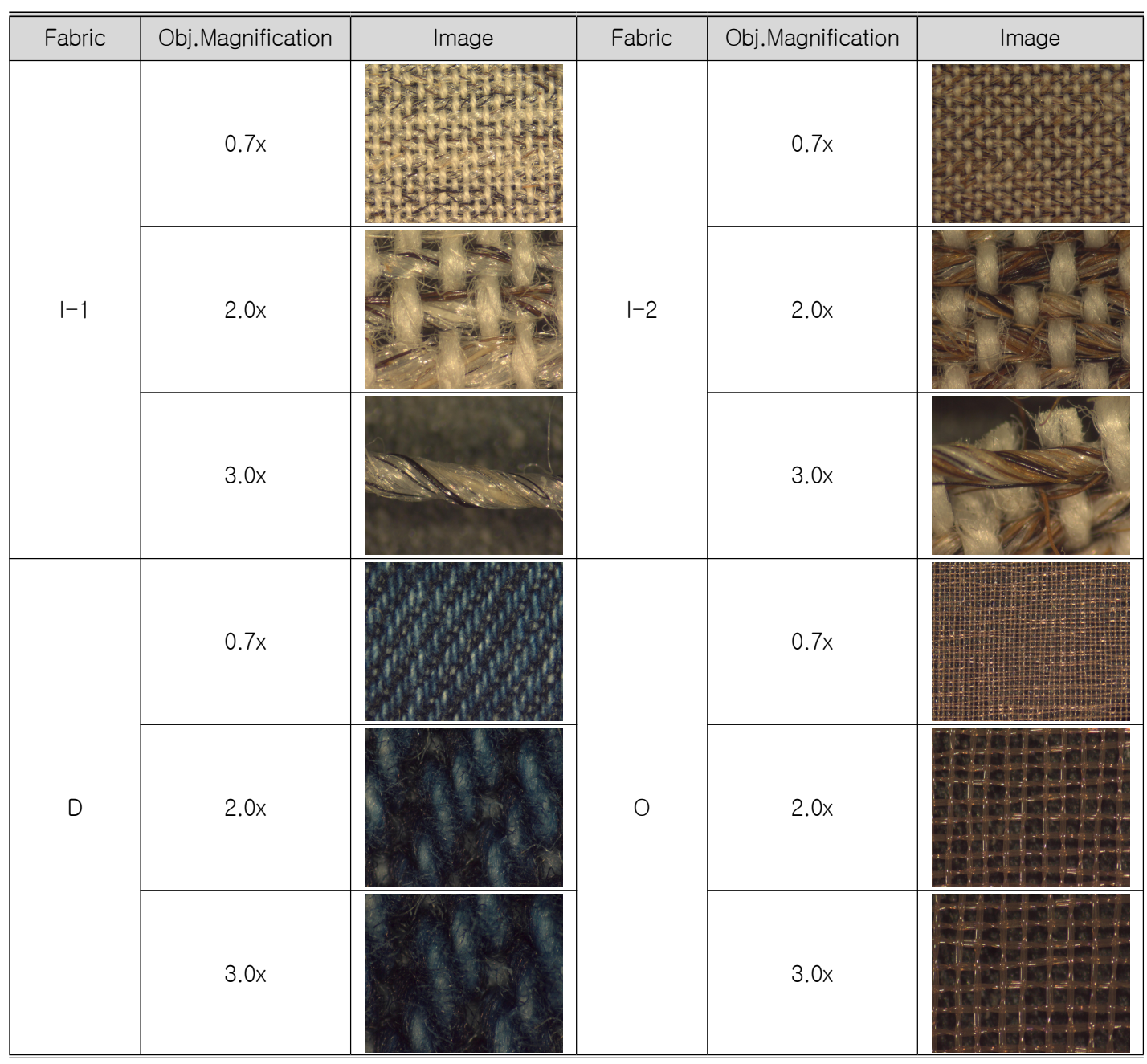


Yoon Jihae $\cdot$ Kim Jongjun / Quilting Effect and Appearance Change according to Fabrics Properties and Surface Reconstruction Method based on 3D Digital Clothing System

shapes are due to the differences in the warp and weft properties of the quilted sleeve specimens, and bending, shear rigidity, thickness, quilting method, polyester wadding, quilting shape, etc. This data were saved in a STL file format and transferred to a mesh surface using Rhino3D. Then, dimensional coordinates were drawn in the sleeve dimensional surface with 'PolylinePoints' of Rhino3D and reconstructed as a chart shown in $<$ Fig. 10>. Height profile were measured as well and saved and expressed in 30 coordinates. Looking at the drape shape generally, it is found that it changes in the order of $|-2>|-1>0>0$. In the case of specimen $\mathrm{O}$, the shape of drape at the bottom shows a unique form that spreads flatter than those of the other specimens. This study measured center profiles only, but other parts' profiles also can be analyzed additionally with the dimensional coordinates, if necessary.

\section{Simulation with 3D Digital Clothing Software System}

Intended patterns were designed using scanned surface image of 4 different fabrics and quilting method of the 30 program. The CLO $3 D$ program simulated the apparel using the property values obtained in this study as shown in <Fig. 11>. According to the observation and comparison result of drape shapes and general silhouette of different materials, 1-2(interlining with large amount of hair) is the most stiff, while $\mathrm{D}$ (Denim) droops the most due to its own weight even with its high bending rigidity. It indicates that drape of quilted outfit is significantly influenced by bending rigidity, weight per unit area, bending length, drape stiffness, shear rigidity, quilting method, and so on.

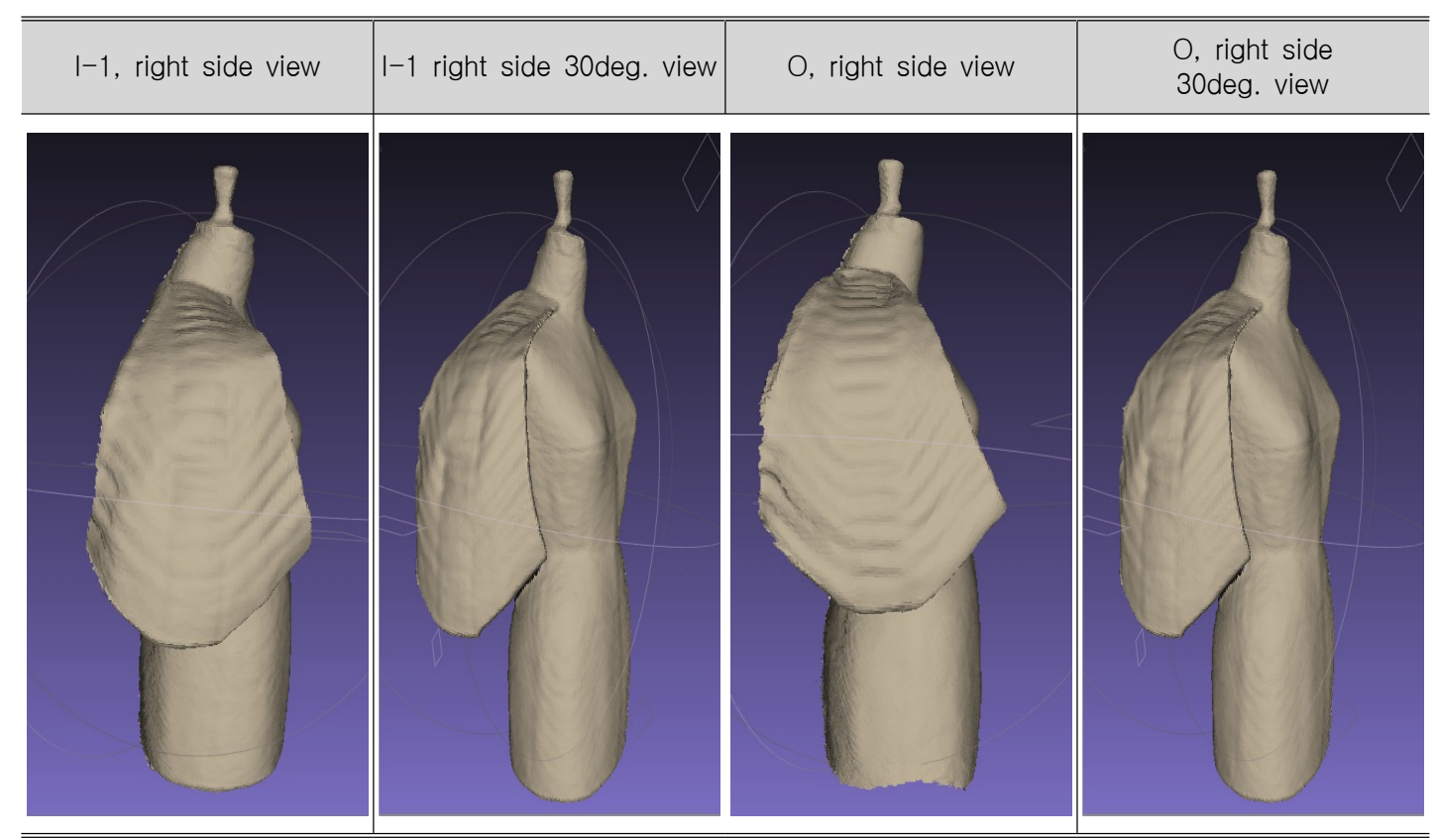

<Fig. 9> 3D Surface Reconstruction Images of Sleeve Specimens: $1-1$ and 0 . 

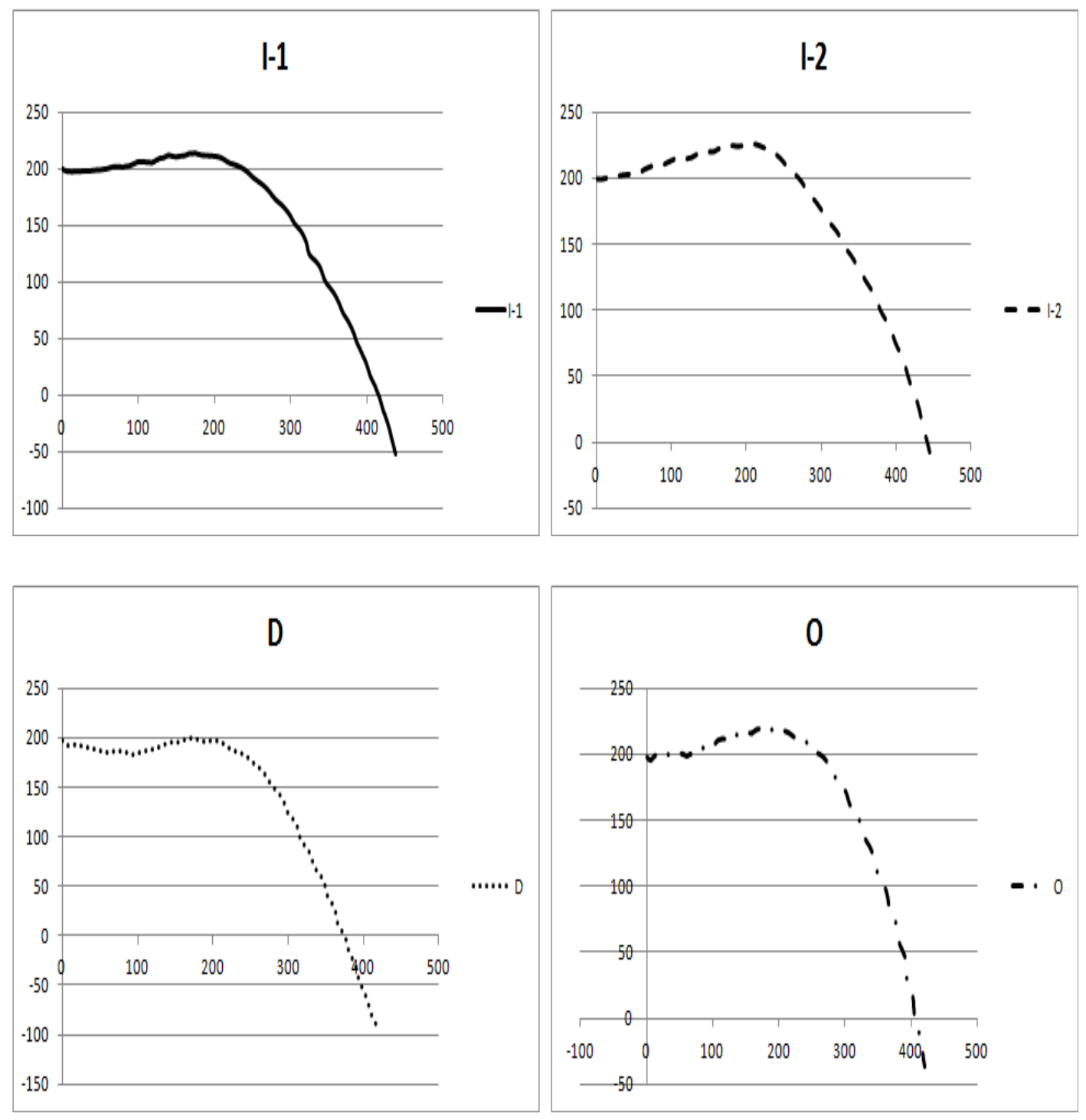

$<$ Fig. 10> Surface profiles of the sleeve specimens mounted on the body.

\section{Conclusion}

Quilting is a technique to join two or more layers of fabrics by stitches or other methods. Interlining is inserted between the fabrics for garment or quilts. In this study, stiff interlining fabrics are used for the face fabric of the garment in order to evaluate the quilting effect. Woven interlining fabrics and other stiff fabrics for comparison purpose are selected to evaluate the effects of the fiber composition, stiffness, or thickness on the appearance of the quilting. Three-dimensional scanning methods are employed to analyze the appearance of the fabrics, 
Yoon Jihae $\cdot$ Kim Jongjun / Quilting Effect and Appearance Change according to Fabrics Properties and Surface Reconstruction Method based on 3D Digital Clothing System

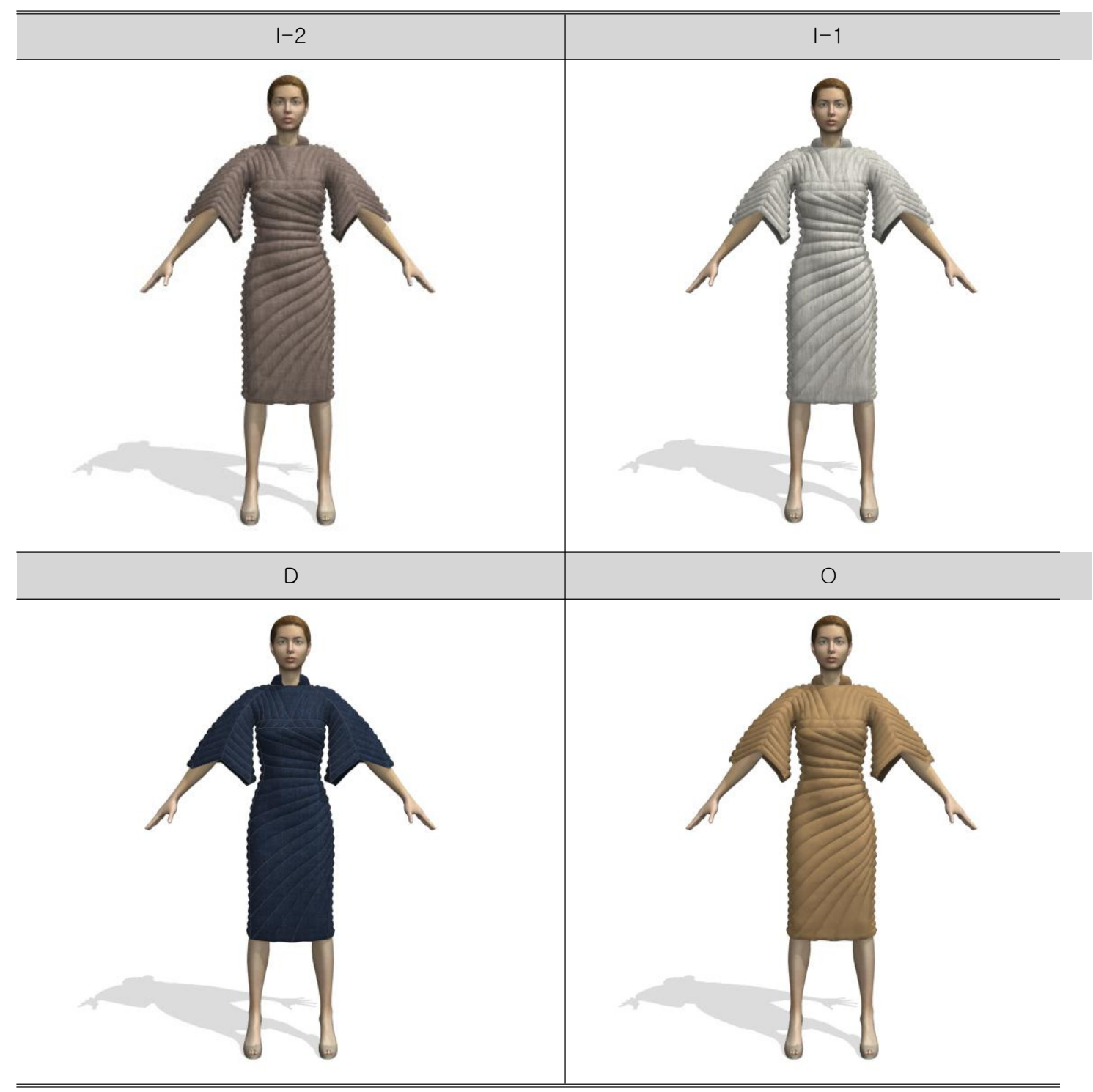

<Fig. 11> Simulated Images using a 3D Digital Clothing CAD System.

stitched fabrics, pad-inserted stitched fabrics, and quilted sleeve fabrics. Currently available 30 digital clothing CAD software system is used to virtually construct the garments made of the interlining fabrics. The results are described as follows:
1. From the Flexometer stiffness test result, the interlining specimen $1-2-2$ shows the highest bending length $(L)$ value. This is due to the fact that the $1-2-2$ has the highest percentage of hair in the filling yarn. Stitched specimens(Type $B$ ) show higher bending length $(L)$ values than their corresponding fabric specimens due to the 
stitches between the layers. Pad-inserted stitched specimens(Type $C$ ) show the highest $L$ values than other two types of specimen types.

2. The values of bending rigidity(B1) of the fabric specimens are in the order of $D>|-1>0>|-2$, and those of $B 2$ are in the order of $|-2>|-1>0>0$. Hair fibers generally show higher bending rigidity than other textile fibers. In order to maximize the stiffness effect, hair fibers and textile fibers are generally spun together for the woven type interlining. Filling yarn of $1-2$ contains $70 \%$ hair, while that of $1-1$ specimen contains $35 \%$. This fact explains the bending rigidity difference of the two specimens. Denim(D) specimen shows highest shear rigidity value.

3. 3D scanning of the testing by Flexometer shows that three-dimensional coordinate values of the fabric specimen describe the overall shape of the bent fabric over the inclined plane of the Flexometer, suggesting the possible use even for the deformed fabric specimen characterization, which is not possible in the conventional testing scheme.

4. 3D scanning and reconstruction of the sleeve specimen installed on the shoulder area of the body provide important information regarding the overall three-dimensional appearance of the quilted sleeve specimen. The information may include bending length, spread of the specimen, etc.

Digital clothing CAD systems have been helping scientists and designers alike to develop the textile and clothing industries in the sectors of apparel manufacturing. As shown in this study, the interlining fabrics may possibly find extended application areas other than the traditional application areas.

We have employed a $3 D$ surface scanning and reconstruction methods in order to express the actual properties and textures of textile materials in the virtual fabric property and appearances depicted in the outcome of the threedimensional digital clothing CAD system. It is expected the employed methods will further be efficiently developed to be utilized in the preparation of $3 \mathrm{D}$ digital clothing.

\section{Reference}

1) Oh, Soon. Kim, Jungsil (2008), A study on the research of the textile pattern design applied by the computer CAD - focusing on the images of dress and its ornament which appeared in the 1960s-, Journal of Korea Society of Design Trend, 20, pp.293-305.

2) Ahn, Hyungjoo (2008), Fashion Design Development based on the Quilting Methods, Ewha Womans University, Graduate School, Master Thesis.

3) Kim, Mikyung (2007), 3D Scanning Techinics and Related Papers, DMC Lab Technical Review, KAIST.

4) Lee, Yoonjoo, Kim, Jongjun, Kim, Kwangyoon, Kim, Kwangil, Bu, Jonghyuk (2011. 4. 30), 3D Clothing Software CLO 3D CAD Application and Suggestions on the Use of Dynamic Displacement of Fashion Fabric Materials, Proc. of 2011 the Korean Society of Fashion Business, pp.62-68.

5) Kawabata, S., Niwa, M.(1991), Objective Measurement of Fabric Mechanical Property and Quality: Its Application to Textile and Clothing Manufacturing, Int. Journal of Clothing Science and Technology, 3(1), pp.7-18.

6) Choi, Kyungme, Kim, Jongjun(2012), A Study on the Tensile Deformation Characteristics 
Yoon Jihae $\cdot$ Kim Jongjun / Quilting Effect and Appearance Change according to Fabrics Properties and Surface Reconstruction Method based on 3D Digital Clothing System

of Knits and Appearance using 3D Virtual Clothing Systems, Journal of Fashion Business, 16(2), pp.151-162.

7) MeshLab Documentation, http://www.cyi.ac.cy/system/files/MeshLab\%2 0Documentation1.pdf, Retrieved on Mar. 11, 2012.,

8) Rhino3D Manual, http://Rhino3D.com/download.htm, Retrieved on Feb. 19, 2012.

9) Kinect, http://www.xbox.com/ko-KR/KINECT, Retrieved on Mar. 7, 2012.

10) ReconstructMe, http://reconstructme.net, Retrieved on Mar. 18, 2012.

11) CLO 3D, http://www.clo3d.com, Retrieved on Apr. 3, 2012.

접수일(2012년 9월 28일),

수정일(1차 : 2012년 10월 22일),

게재확정일(2012년 10월 31일) 